\title{
Qa-1 restriction of CD8+ suppressor T cells
}

\author{
Stefanie Sarantopoulos, 1,2,3 Linrong Lu, ${ }^{1,4}$ and Harvey Cantor ${ }^{1,4}$
}

\author{
1Department of Cancer Immunology and AIDS, ${ }^{2}$ Department of Medical Oncology, Dana Farber Cancer Institute, Boston, Massachusetts, USA \\ ${ }^{3}$ Department of Medicine, Brigham and Women's Hospital, Boston, Massachusetts, USA. ${ }^{4}$ Department of Pathology, \\ Harvard Medical School, Boston, Massachusetts, USA.
}

\begin{abstract}
There is increasing evidence that the immune response can be inhibited by several $\mathrm{T}$ cell subsets, including NK $\mathrm{T}$ cells, $\mathrm{CD25}^{+} \mathrm{CD}^{+} \mathrm{T}$ cells, and a subpopulation of $\mathrm{CD8}^{+} \mathrm{T}$ cells. Animal model studies of multiple sclerosis have suggested an important role for suppressor $\mathrm{CD8}^{+} \mathrm{T}$ cells in protection against disease recurrence and exacerbation. The molecular lynchpin of $\mathrm{CD8}^{+}$suppressive activity is the murine MHC molecule Qa-1, termed HLA-E in humans. Here we summarize findings from work on Qa-1 that have begun to delineate suppressor $\mathrm{CD8}^{+} \mathrm{T}$ cells and their mechanisms of action in the context of self tolerance and autoimmune disease.
\end{abstract}

\section{Mechanisms of peripheral tolerance}

The first stages of $\mathrm{T}$ cell development occur in the thymus, where a selection process weeds out potentially autoreactive cells. Successful progression of thymocytes through selection requires expression of TCRs capable of efficient binding to self-MHC products. Although $\mathrm{T}$ cell clones bearing TCRs with high affinity for selfpeptide MHC products are generally eliminated during this process, the resulting $\mathrm{T}$ cell repertoire is still strongly biased toward self reactivity (1). As a result, substantial numbers of peripheral $\mathrm{T}$ cells can proliferate in response to self-peptide MHC complexes, and some can differentiate into effector cells in the context of inflammatory stimuli (2-4).

Expansion of autoreactive $T$ cells in peripheral lymphoid tissues is constrained, in part, by abortive TCR signals that lead to T cell elimination or inactivation $(5,6)$. However, mechanisms of T cell elimination that include activation-induced cell death (AICD) and anergy, an insensitivity to antigens that creates an inability to elicit a normal antigenic response, may not suffice to prevent autoimmune disease $(7,8)$. The apparent limitations of these mechanisms for eliminating all self-reactive cells have stimulated research into $\mathrm{T}$ cells and other cell types, so-called suppressor $\mathrm{T}$ cells, that may exert dominant inhibitory effects on expansion of pathogenic autoreactive T cells. One such group of cells is the suppressor $\mathrm{CD}^{+} \mathrm{T}$ cells.

\section{Suppressor CD8+ T cells and Qa-1}

The recent resurgence of interest in suppressor $\mathrm{T}$ cells has been largely due to the delineation of a $\mathrm{CD}^{+}$sublineage with regulatory activity (9-13). The possibility that $\mathrm{CD}^{+} \mathrm{T}$ cells might also contain a regulatory sublineage has received much less attention despite the fact that $\mathrm{CD}^{+} \mathrm{T}$ cells were the initial target of work in this area. Early research used experimental systems that mainly measured in vitro antibody responses to complex antigens such as foreign erythrocytes as well as in vivo contact hypersensitivity and delayed-typehypersensitivity reactions. Downregulation of these responses by subpopulations of $\mathrm{CD}^{+} \mathrm{T}$ cells was then difficult to distinguish from modes of inhibition that were not yet well understood, includ-

Nonstandard abbreviations used: AICD, activation-induced cell death; EAE, experimental autoimmune encephalomyelitis; hsp, heat shock protein; $\beta_{2} \mathrm{~m}, \beta_{2}$ microglobulin; MBP, myelin basic protein; PLP, proteolipid protein; Qdm, Qa-1 determinant modifiers; TAP, transporter associated with antigen processing.

Conflict of interest: The authors have declared that no conflict of interest exists.

Citation for this article: J. Clin. Invest. 114:1218-1221 (2004).

doi:10.1172/JCI200423152. ing the impact of regulatory cytokines and AICD on $\mathrm{CD}^{+} \mathrm{T}$ cells. These difficulties, combined with the inability of molecular genetics to validate early models by identifying genes for suppressor factors and receptors, gave rise to a well-justified skepticism concerning the existence of suppressor $\mathrm{CD}^{+} \mathrm{T}$ cells. However, some useful information can be gleaned by reexamining these early approaches. For example, the ability of $\mathrm{T}$ cell vaccination with pathogenic autoreactive $\mathrm{CD} 4^{+}$clones to inhibit autoimmune disease $(14,15)$ has been verified, along with suggestions that protection depends on induction of suppressor $\mathrm{CD}^{+} \mathrm{T}$ cells $(16-18)$.

\section{Murine Qa-1}

More recent studies have suggested that a subpopulation of $\mathrm{CD}^{+}$ T cells might suppress the response of successfully activated CD $4^{+}$ $T$ cells and $B$ cells through an interaction that depends on expression by target cells (or APCs) of the HLA class Ib MHC molecule Qa-1 $(19,20)$. The Qa-1 protein is a member of the class Ib MHC family. Most class Ib MHC gene products interact with CD8 on the surface of $\mathrm{T}$ cells and are expressed on the cell surface along with $\beta_{2}$ microglobulin $\left(\beta_{2} \mathrm{~m}\right)$ molecules. Several class Ib molecules are ligands for receptors on NK cells, e.g., Qa-1 is a ligand for the inhibitory CD94/NKG2A or CD94/NKG2C receptors.

The impact of molecular immunology on our understanding of Tregs is evident from research into the murine Qa-1 protein. Early work revealed an inhibitory interaction between $\mathrm{CD}^{+}$and $\mathrm{CD}^{+} \mathrm{T}$ cells in vitro that depended on the expression of the Qa-1 molecule on activated $\mathrm{CD}^{+}$target cells (21). The significance of this observation became clearer after Qa-1 was shown to be a peptide-binding class Ib molecule (rather than an activation-related surface protein); it mapped to the $3^{\prime}$ end of the MHC class I region on chromosome 17 , the same region that contained other class Ib genes, including thymus leukemia antigen (22-24). The mouse MHC class Ib molecule Qa-1 and its human homologue, the HLA-E protein, form heterodimers with $\beta_{2} \mathrm{~m}$ in lymphoid cells that bind to and present peptides derived from self or foreign proteins (25-28). Although Qa-1 RNA is expressed in many cell types (29), surface expression of the Qa-1- $\beta_{2}$ m heterodimer is highly constrained by Qa- $1-\beta_{2} \mathrm{~m}$ assembly and transport mechanisms that favor expression on activated $\mathrm{T}$ and $\mathrm{B}$ lymphocytes and dendritic cells $(28,30)$.

\section{Qa-1 gene products engage two classes of receptors}

Peptide-containing Qa-1 complexes engage two classes of receptors. Qa-1 heterodimers containing peptides derived in a transporter associated with antigen processing-dependent (TAP-dependent) 


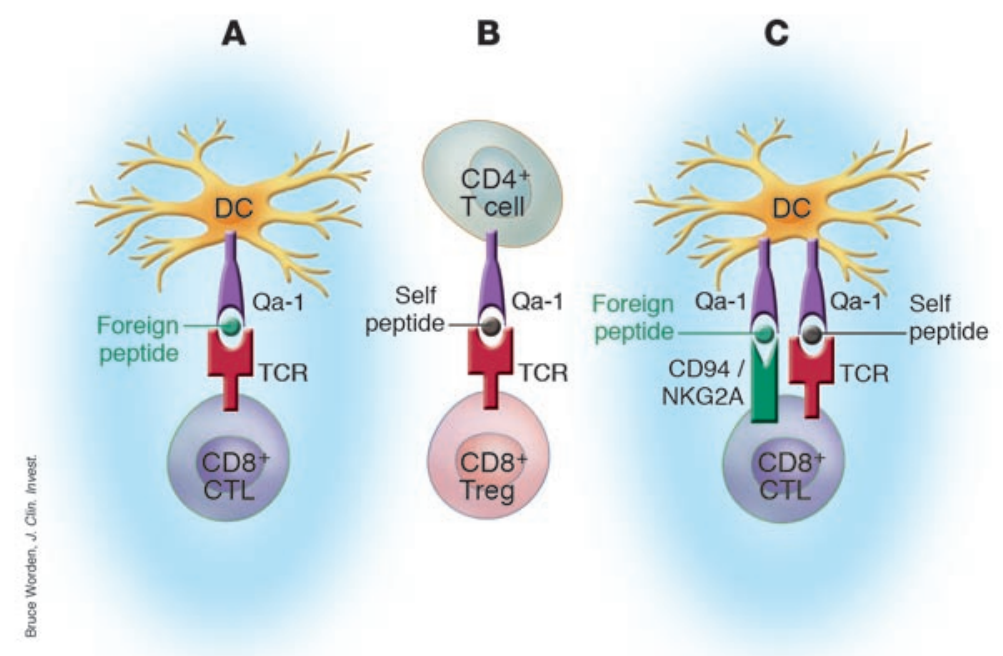

Figure 1

Engagement of Qa-1 by the TCR and by CD94/NKG2A. (A) Presentation of Qa-1bacterial GroEL peptide by a DC following Salmonella infection to $C D 8^{+} \mathrm{T}$ cells, where the receptor is a TCR, leads to CTL responses. (B) Presentation of Qa-1-self peptides by activated $\mathrm{CD}^{+}{ }^{+} \mathrm{T}$ cells to $\mathrm{CD} 8^{+} \mathrm{T}$ cells, where the receptor is a TCR, leads to the development of Qa-1-restricted suppressor CD8 ${ }^{+} \mathrm{T}$ cells. (C) Engagement of CD94/NKG2A receptors on CD8 ${ }^{+} \mathrm{T}$ cells with a DC can inhibit either TCR-mediated CTL responses specific for Qa-1-foreign peptide ligands and/or TCR-mediated suppressive responses specific for Qa-1-self peptide ligands. This NKG2A-dependent interaction may regulate expression of suppressor or cytotoxic $\mathrm{CD} 8^{+} \mathrm{T}$ cells through inhibition of cellular activation or diminished AICD.

fashion from class Ia leader sequences, termed Qa-1 determinant modifiers (Qdm), bind to nonclonally distributed CD94-NKG2A, $\mathrm{C}$, and/or E receptors on NK cells and a subpopulation of CD8 cells. The functional outcome of Qdm:NKG2 interactions is generally inhibition of NK or CD8 CTL activity (31). A second class of Qa-1 ligands is comprised of Qa-1: $\beta_{2} \mathrm{~m}$ heterodimers containing a growing number of peptides, including those derived from TCR $V_{\beta}$, preproinsulin, bacterial GroEL, and endogenous heat shock protein 60 (hsp60) (27, 32-34). Interaction between this class of Qa-1 ligands and the TCR on CD8 cells can promote CD8 cell activation, expansion, and expression of effector cell activity (35).

\section{Qa-1-restricted suppression by CD8+ $\mathrm{T}$ cells}

As noted above, interest in $\mathrm{CD} 8^{+} \mathrm{T}$ cell suppression has been rekindled by studies of experimental autoimmune encephalomyelitis (EAE) in B10.PL mice, which become resistant to disease after initial exposure to myelin basic protein (MBP). Mice deficient in $\mathrm{CD}^{+}$ $\mathrm{T}$ cells due to a targeted mutation or antibody-dependent depletion do not develop resistance to $\operatorname{EAE}(36,37)$. Resistance requires exposure of $\mathrm{CD}^{+} \mathrm{T}$ cells to $\mathrm{MBP}$, since replenishment of $\mathrm{CD} 8^{+}-$ deficient mice with $\mathrm{CD}^{+} \mathrm{T}$ cells from nonimmune donors before a secondary anti-MBP response does not prevent disease recurrence. Additional studies by Jiang, Chess, and colleagues suggest that the inhibitory interaction between $\mathrm{CD}^{+}$Tregs and MBP-reactive $\mathrm{CD} 4^{+}$ $\mathrm{T}$ cells involves TCR-based recognition of Qa-1-self-peptide complexes expressed by autoreactive $\mathrm{CD}^{+} \mathrm{T}$ cells $(16,17,35)$.

To develop a clearer understanding of the physiological role of Qa-1-reactive CD8 ${ }^{+} \mathrm{T}$ cells in suppressing immune responses, we recently generated mice that are deficient in Qa-1 gene expression due to a targeted gene mutation (38). Qa-1-deficient animals devel- op exaggerated secondary $\mathrm{CD}^{+} \mathrm{T}$ cell responses after viral infection or immunization with foreign or self peptides. In addition, enhanced $\mathrm{CD} 4^{+} \mathrm{T}$ cell responses of Qa-1-deficient mice to mouse proteolipid protein (PLP) self peptide are associated with increased susceptibility to recurrence of EAE; this phenotype is remedied by lentiviral-based expression of the correct Qa-1 allele (38). These data indicate Qa-1 is required for an appropriately curtailed $\mathrm{CD}^{+} \mathrm{T}$ cell response to infection and for autoimmunity resistance.

Analysis of the cellular basis of Qa-1-dependent resistance to EAE indicated that $\mathrm{CD}^{+} \mathrm{T}$ cells from PLP-peptide immune mice suppressed peptide-specific expansion of $\mathrm{CD}^{+} \mathrm{T}$ cells; both EAE resistance and the development of suppressor $\mathrm{CD}^{+} \mathrm{T}$ cells required prior immunization with PLP peptide. These findings indicate that the primary immune response to self peptides may include expansion of Qa-1restricted $\mathrm{CD}^{+} \mathrm{T}$ cells that can inhibit expansion of pathogenic autoreactive $\mathrm{CD} 4^{+} \mathrm{T}$ cells and consequent autoimmune disease. The impact of $\mathrm{CD}^{+}$-dependent suppression on the clonal composition of MBP-reactive $\mathrm{CD} 4^{+} \mathrm{T}$ cells has been ascertained through complementarity-determining region 3 sequence analysis, a PCR-based technique that allows amplification and definition of distinct antigen-binding regions of TCR chains, of $\mathrm{CD}^{+} \mathrm{T}$ cells after recovery from EAE in the presence or absence of $\mathrm{CD}^{+} \mathrm{T}$ cells. Depletion of $\mathrm{CD}^{+} \mathrm{T}$ cells was associated with expansion of several dominant clones of pathogenic autoreactive $\mathrm{CD} 4^{+} \mathrm{T}$ cells in contrast to the more heterogeneous TCR repertoire of $\mathrm{CD} 4^{+}$ T cells, each of limited clonal expansion, that developed after exposure to MBP in the presence of $\mathrm{CD}^{+} \mathrm{T}$ cells (35).

Analysis of Qa-1-restricted suppression suggests that expression of a TCR on $\mathrm{CD}^{+}$suppressor T cells may be necessary for recognition of expression of suppressive activity (38). Another observation consistent with this idea is that constraints placed on expression of a full TCR repertoire prevent CD8 cells from mediating suppression. Changes in the structure of Qa-1 on target cells that may affect peptide binding also affect susceptibility to suppression of CD8 cells. $\mathrm{CD}^{+} \mathrm{T}$ cells expressing an incorrect TCR transgene are unable to mediate suppressive activity (38). This finding is consistent with TCR-dependent discrimination of peptide-Qa-1 complexes on target $\mathrm{CD}^{+} \mathrm{T}$ cells. NKG2-dependent recognition of Qa-1, on the other hand, would not be expected to be affected by allele-specific changes, although without additional analysis of Qa-1 proteins that express defined mutations, this remains uncertain. Since NKG2 receptors on $\mathrm{CD}^{+} \mathrm{T}$ cells may affect the levels of TCR-dependent suppressive activity, e.g., through regulation of TCR-dependent activation and expansion of these cells (Figure 1), experimental analysis using cells that express mutant Qa-1 molecules that differentially engage the TCR and NKG2 receptors is needed for a full molecular dissection of the contribution of these two Qa-1-binding recognition elements to suppressive $\mathrm{CD}^{+}$activity.

The timing of $\mathrm{CD}^{+}$-dependent inhibitory responses can be contrasted with that of the naturally occurring $\mathrm{CD} 4^{+} \mathrm{CD} 25^{+}$Tregs, which interrupt expansion of self-reactive $\mathrm{T}$ cells during the initial stages of primary responses. Suppressor $\mathrm{CD} 8^{+} \mathrm{T}$ cells arise later in the immune response and become apparent experimentally only after restimulation by antigen or superantigen $(11,38,39)$. The development of Qa-1- 
restricted suppressive activity more closely resembles the kinetics of $\mathrm{CD} 8^{+}$-dependent cytolytic activity rather than $\mathrm{CD} 4{ }^{+} \mathrm{CD} 25^{+}$regulatory activity. Both $\mathrm{CD}^{+}$-dependent suppressive and cytolytic responses require primary immunization to generate TCR-dependent lysis or Qa-1-restricted elimination of target cells $(38,39)$.

The mechanism of Qa-1-restricted inhibitory interactions has not been established. $\mathrm{CD}^{+}$cells that recognize Qa-1 associated with insulin peptide efficiently kill Qa-1+ ${ }^{+}$lymphoblastoid target cells in the presence of intact soluble insulin after TAP-independent processing and presentation of insulin-derived peptide by Qa-1 (33). Qa-1-restricted inhibitory activity of CD8 ${ }^{+} \mathrm{T}$ cells may reflect lysis of activated $\mathrm{CD}^{+} \mathrm{T}$ cells that display Qa-1-peptide complexes, perhaps after TCR-dependent signals induce expression of relevant Qa-1 ligands on activated T and B lymphocytes. Although the Qa-1-binding peptides that target $\mathrm{CD}^{+}$responses have not been fully identified, they may represent a relatively restricted set that is efficiently expressed on activated $\mathrm{T}$ cells after high avidity TCR-ligand engagement (35). The fate of individual TCRs following ligation with peptide-MHC complexes is thought to depend on peptide affinity, the association/dissociation rate of TCR-ligand formation, and the strength of TCR-dependent signals. Most internalized TCR complexes are degraded in lysosomes (40), but robust TCR engagement may facilitate a proteosomedependent degradation process that leads to recycling and TAPindependent Qa-1 presentation (41).

\section{Peptide presentation and selection of $\mathrm{CD}^{+} \mathrm{T}$ cells by $\mathrm{Qa}-1$}

Positive selection of thymocytes that express a TCR transgene specific for foreign peptides associated with Qa-1 depends on interactions with Qa-1 peptides and self peptides expressed primarily by hematopoietic cells (42). Both selection and antigen recognition are TCR-dependent and do not require NKG2 receptors (28). Whether Qa-1-dependent negative selection by self peptides is dependent on TCR engagement or other receptors is unclear. A bacterial GroEL peptide that is almost identical to murine hsp60 peptide (GroEL: GMQFDRGYL; murine hsp60: GMKFDRGYI) elicits cytolytic CD8 ${ }^{+}$ $\mathrm{T}$ cells that are cross-reactive with the murine hsp60 self peptide. One explanation for high levels of Qa-1-restricted reactivity to this self peptide is that negative selection by Qa-1 is less efficient than negative selection by conventional class I MHC. Alternatively, expression of CD8 reactivity to Qa-1-restricted self peptides may reflect defects in peripheral tolerance mechanisms.

The expression, structure, and processing pathways of Qa-1 are distinct from those of classical MHC class I. Class Ia molecules are widely expressed on most cell types regardless of activation status. Qa-1 is preferentially expressed on activated but not resting $\mathrm{T}$ cells and thymocytes, thus protecting unstimulated $\mathrm{T}$ cells from the effects of $\mathrm{CD}^{+}$-dependent suppression. Moreover, Qa-1 expression on activated $\mathrm{CD}^{+} \mathrm{T}$ cells is short-lived, and optimal levels persist for hours rather than days after TCR ligation. Susceptibility of activated $\mathrm{CD}^{+} \mathrm{T}$ cells to suppression by $\mathrm{CD}^{+} \mathrm{T}$ cells is thus restricted to a brief window after immunization. Class Ia molecules are the most highly polymorphic human or mouse genes studied to date, but Qa-1 has very limited polymorphisms and has very tight specificity of peptide binding. Qa-1 was first found to bind the Qdm peptide; subsequent studies have shown that this class Ib MHC molecule is capable of presenting a variety of self and foreign peptides to $\mathrm{CD}^{+} \mathrm{T}$ cells through TAP-dependent and TAP-independent pathways. The latter pathway allows
Qa-1 on dendritic cells to cross-present peptides to $\mathrm{CD}^{+} \mathrm{T}$ cells, and similar trafficking machinery may allow Qa-1-dependent cross-presentation of peptides by activated $\mathrm{CD}^{+} \mathrm{T}$ cells. If so, this mechanism would allow the presentation of self peptides derived from the TCR or hsp60 in activated $\mathrm{CD}^{+} \mathrm{T}$ cells to $\mathrm{CD} 8^{+} \mathrm{T}$ cells. The observation that activated lymphoid cells can efficiently process and present intact exogenous insulin associated with Qa-1 is consistent with this mechanism. Findings that engagement of the TCR by peptide ligands can lead to endocytosis of the TCR also suggest a mechanism that may allow TAP-independent endosomal loading of TCR-derived peptides onto Qa-1 molecules.

What about the contribution of non-TCR receptors to Qa-1restricted activation of $\mathrm{CD}^{+} \mathrm{T}$ cells? Although CD94/NKG2 receptors are expressed by less than $2.5 \%$ of $\mathrm{CD}^{+} \mathrm{T}$ cells in humans (43), they are upregulated after TCR engagement or stimulation by cytokines, including TGF- $\beta$ and IL-15 $(44,45)$. CD94/NKG2A expression is also induced shortly after $\mathrm{T}$ cell activation following lymphocytic choriomeningitis or Listeria monocytogenes infection. Regulatory $\mathrm{CD}^{+} \mathrm{T}$ cells may express both clonally distributed TCR $\alpha \beta$ receptors for Qa-1-self peptide $(19,20,35)$ and nonclonally distributed NKG2 ${ }^{+}$coreceptors for Qdm-Qa-1 (46). Interactions between Qa-1-peptide ligands and the TCR $\alpha \beta$ receptor may stimulate clonal expansion of $\mathrm{CD}^{+} \mathrm{T}$ cells and development of suppressive or effector activity, while interactions between Qa-1-Qdm and CD94-NKG2 receptors on $\mathrm{CD}^{+} \mathrm{T}$ cells may downregulate expansion and/or protect against $\operatorname{AICD}(32,47)$ (see Figure 1). According to this model, differential engagement of these two classes of Qa-1 receptors may regulate the expansion and activation of suppressor $\mathrm{CD}^{+} \mathrm{T}$ cells.

\section{$\mathrm{CD}^{+} \mathrm{T}$ cell suppression and self tolerance}

The potential role of $\mathrm{Qa}-1$-restricted $\mathrm{CD}^{+} \mathrm{T}$ cells in clinical or animal models - aside from murine EAE - has not been investigated. However, experimental analysis of other animal models of autoimmune disease and organ transplantation have suggested a potential role for inhibitory $\mathrm{CD}^{+} \mathrm{T}$ cells and/or $\mathrm{T}$ cells that may recognize conserved Qa-1-peptide ligands. Vaccination with self or foreign hsp60 can inhibit development of adjuvant-induced arthritis, and transfer of hsp65 peptide-primed $\mathrm{T}$ cells can afford protection against disease development in adoptive hosts $(48,49)$. Analysis of the protective activity of hsp-derived peptides in murine adjuvant-induced arthritis (50) and in some mouse models of type 1 diabetes (51) in animals deficient in Qa-1 expression may help to clarify the contribution of Qa-1-restricted CD8 ${ }^{+} \mathrm{T}$ cells to disease protection.

Studies of transplantation tolerance have also suggested a possible role for suppressor $\mathrm{CD}^{+} \mathrm{T}$ cells. Early experiments first suggested that Qa-1-specific transplantation tolerance could prevent generation of alloreactive CTL (52) as well as the generation of suppressor $\mathrm{T}$ cell activity in anti-Qa-1 immune responses $(53,54)$. $\mathrm{CD}^{+} \mathrm{T}$ cells may contribute to the inhibitory effects of pretransplant donor-specific blood transfusion since elimination of this subset before blood transfusion can result in graft rejection (55). The potential role of Qa-1/HLA-E-restricted CD8 cells in these responses is not currently understood.

\section{Concluding remarks}

Definition of the role of $\mathrm{CD}^{+} \mathrm{T}$ cells that suppress the response of $\mathrm{CD}^{+} \mathrm{T}$ cells expressing Qa-1-peptide target ligands has important implications for understanding immunoregulation in the context of self tolerance. Further investigation of animal models of arthritis, autoimmune type I diabetes, and organ transplantation is needed to 
delineate the regulatory role of the Qa-1/HLA-E-restricted mechanisms defined in this review. The availability of Qa-1 deficient mice represents a new experimental approach to evaluation of the potential role of Qa-1-restricted suppression in these disorders.
Address correspondence to: Harvey Cantor, Dana Farber Cancer Institute, 44 Binney Street, Boston, Massachusetts 02115, USA. Phone: (617) 632-3348; Fax: (617) 632-4630; E-mail: Harvey_Cantor@dfci.harvard.edu.
1. Cantor, H. 2000. T-cell receptor crossreactivity and autoimmune disease. Adv. Immunol. 75:209-233.

2. Goldrath, A.W., and Bevan, M.J. 1999. Selecting and maintaining a diverse T-cell repertoire. Nature. 402:255-262.

3. Bouneaud, C., Kourilsky, P., and Bousso, P. 2000. Impact of negative selection on the $T$ cell repertoire reactive to a self-peptide: a large fraction of $\mathrm{T}$ cell clones escapes clonal deletion. Immunity. 13:829-840.

4. Slifka, M.K., et al. 2003. Preferential escape of subdominant CD8+ T cells during negative selection results in an altered antiviral $\mathrm{T}$ cell hierarchy. J. Immunol. 170:1231-1239.

5. Kearney, E.R., Pape, K.A., Loh, D.Y., and Jenkins, M.K. 1994. Visualization of peptide-specific T cell immunity and peripheral tolerance induction in vivo. Immunity. 1:327-339.

6. Martin, D.A., et al. 1999. Defective CD95/APO-1/ Fas signal complex formation in the human autoimmune lymphoproliferative syndrome, type Ia. Proc. Natl. Acad. Sci. U. S. A. 96:4552-4557.

7. Anderton, S.M., Radu, C.G., Lowrey, P.A., Ward, E.S., and Wraith, D.C. 2001. Negative selection during the peripheral immune response to antigen. J. Exp. Med. 193:1-11.

8. Panoutsakopoulou, V., et al. 2001. Analysis of the relationship between viral infection and autoimmune disease. Immunity. 15:137-147.

9. Wood, K.J., and Sakaguchi, S. 2003. Regulatory $\mathrm{T}$ cells in transplantation tolerance. Nat. Rev. Immunol. 3:199-210.

10. Shevach, E.M. 2002. CD4+ CD25+ suppressor T cells: more questions than answers. Nat. Rev. Immunol. 2:389-400.

11. Sakaguchi, S., et al. 2001. Immunologic tolerance maintained by CD25+ CD4+ regulatory T cells: their common role in controlling autoimmunity, tumor immunity, and transplantation tolerance. Immunol. Rev. 182:18-32.

12. Apostolou, I., Sarukhan, A., Klein, L., and von Boehmer, H. 2002. Origin of regulatory T cells with known specificity for antigen. Nat. Immunol. 3:756-763.

13. Gavin, M., and Rudensky, A. 2003. Control of immune homeostasis by naturally arising regulatory CD4(+) T cells. Curr. Opin. Immunol. 15:690-696.

14. Ben-Nun, A., Wekerle, H., and Cohen, I.R. 1981. Vaccination against autoimmune encephalomyelitis with T-lymphocyte line cells reactive against myelin basic protein. Nature. 292:60-61.

15. Cohen, I.R., and Weiner, H.L. 1988. T cell vaccination. Immunol. Today. 9:332-335.

16. Jiang, H., et al. 1998. T cell vaccination induces TCR V $\beta$ specific, Qa-1 restricted regulatory CD8+ T cells. Proc. Natl. Acad. Sci. U. S. A. 95:4533-4537.

17. Jiang, H., Braunstein, N.S., Yu, B., Winchester, R., and Chess, L. 2001. CD8+ T cells control the TH phenotype of MBP-reactive CD4+ T cells in EAE mice. Proc. Natl. Acad. Sci. U. S. A. 98:6301-6306.

18. Panoutsakopoulou, V., et al. 2004. Suppression of autoimmune disease after vaccination with autoreactive T cells that express Qa-1 peptide complexes. J. Clin. Invest. 113:1218-1224. doi:10.1172/ JCI200420772.

19. Noble, A., Zhao, Z.-S., and Cantor, H. 1998. Suppression of immune responses by CD8 cells: II. Qa-1 on activated B-cells stimulates CD8 cell suppression of Th2-dependent antibody responses. J. Immunol. 160:566-571.

20. Jiang, H., and Chess, L. 2000. The specific regulation of immune responses by CD8+ T cells restrict- ed by the MHC class Ib molecule, Qa-1. Annu. Rev. Immunol. 18:185-216.

21. Cantor, H., et al. 1978. Immunoregulatory circuits among $\mathrm{T}$ cell sets. Identification of a subpopulation of T-helper cells that induces feedback inhibition. J. Exp. Med. 148:871-877.

22. Stanton, T.H., and Boyse, E.A. 1976. A new serologically defined locus, Qa-1, in the Tla-region of the mouse. Immunogenetics. 3:525-531.

23. Lalanne, J.L., et al. 1985. Expression of class I genes in the major histocompatibility complex: identification of eight distinct mRNAs in DBA/ 2 mouse liver. Cell. 41:469-478.

24. Connolly, D.J., et al. 1993. A cDNA clone encoding the mouse Qa-1a histocompatibility antigen and proposed structure of the putative peptide binding site. J. Immunol. 151:6089-6098.

25. Aldrich, C.J., et al. 1992. T cell recognition of QA$1 \mathrm{~b}$ antigens on cells lacking a functional Tap-2 transporter. J. Immunol. 149:3773-3777.

26. Lo, W.F., Ong, H., Metcalf, E.S., and Soloski, M.J. 1999. T cell responses to gram-negative intracellular bacterial pathogens: a role for CD8+ $\mathrm{T}$ cells in immunity to Salmonella infection and the involvement of MHC class Ib molecules. J. Immunol. 162:5398-5406.

27. Lo, W.F., et al. 2000. Molecular mimicry mediated by MHC class Ib molecules after infection with gram-negative pathogens. Nat. Med. 6:215-218.

28. Sullivan, B.A., Kraj, P., Weber, D.A., Ignatowicz, L., and Jensen, P.E. 2002. Positive selection of a Qa-1-restricted T cell receptor with specificity for insulin. Immunity. 17:95-105.

29. Transy, C., et al. 1987. A low polymorphic mouse H-2 class I gene from the Tla complex is expressed in a broad variety of cell types. J. Exp. Med. 166:341-361.

30. Soloski, M.J., DeCloux, A., Aldrich, C.J., and Forman, J. 1995. Structural and functional characteristics of the class IB molecule, Qa-1. Immunol. Rev. 147:67-89.

31. Moser,J.M., Gibbs, J., Jensen, P.E., and Lukacher, A.E. 2002. CD94-NKG2A receptors regulate antiviral CD8(+) T cell responses. Nat. Immunol. 3:189-195.

32. Davies, A., et al. 2003. A peptide from heat shock protein 60 is the dominant peptide bound to Qa-1 in the absence of the MHC class Ia leader sequence peptide Qdm. J. Immunol. 170:5027-5033.

33. Tompkins, S.M., Kraft, J.R., Dao, C.T., Soloski, M.J., and Jensen, P.E. 1998. Transporters associated with antigen processing (TAP)-independent presentation of soluble insulin to alpha/beta $\mathrm{T}$ cells by the class Ib gene product, Qa-1(b). J. Exp. Med. 188:961-971.

34. Seaman, M.S., Perarnau, B., Lindahl, K.F., Lemonnier, F.A., and Forman, J. 1999. Response to Listeria monocytogenes in mice lacking MHC class Ia molecules. J. Immunol. 162:5429-5436.

35. Jiang, H., et al. 2003. Regulatory CD8+ T cells finetune the myelin basic protein-reactive $\mathrm{T}$ cell receptor $\mathrm{V}$ beta repertoire during experimental autoimmune encephalomyelitis. Proc. Natl. Acad. Sci.U.S. A. 100:8378-8383.

36. Jiang, H., Zhang, S.-L., and Pernis, B. 1992. Role of CD8+ T cells in murine experimental allergic encephalomyelitis. Science. 256:1213-1215.

37. Koh, D.R., et al. 1992. Less mortality but more relapses in experimental allergic encephalomyelitis in CD8-/- mice. Science. 256:1210-1213.

38. Hu, D., et al. 2004. Analysis of regulatory CD8 cells in mice deficient in the Qa-1 class Ib molecule. Nat. Immunol. 5:516-523.

39. Jiang, H., and Chess, L. 2004. An integrated model of immunoregulation mediated by regulatory $\mathrm{T}$ cell subsets. Adv. Immunol. 83:253-288.

40. Valitutti, S., Muller, S., Salio, M., and Lanzavecchia, A. 1997. Degradation of T cell receptor (TCR)-CD3zeta complexes after antigenic stimulation. J. Exp. Med. 185:1859-1864.

41. Liu, H., Rhodes, M., Wiest, D.L., and Vignali, D.A. 2000. On the dynamics of TCR:CD3 complex cell surface expression and downmodulation. Immunity. 13:665-675.

42. Urdahl, K.B., Sun, J.C., and Bevan, M.J. 2002. Positive selection of MHC class Ib-restricted CD8(+) T cells on hematopoietic cells. Nat. Immunol. 3:772-779.

43. Speiser, D.E., et al. 1999. CD28-negative cytolytic effector $T$ cells frequently express NK receptors and are present at variable proportions in circulating lymphocytes from healthy donors and melanoma patients. Eur. J. Immunol. 29:1990-1999.

44. Mingari, M.C., et al. 1998. HLA class I-specific inhibitory receptors in human $\mathrm{T}$ lymphocytes: interleukin 15-induced expression of CD94/NKG2A in superantigen- or alloantigen-activated CD8+ T cells. Proc. Natl. Acad. Sci. U. S. A. 95:1172-1177.

45. Bertone, S., et al. 1999. Transforming growth factor-beta-induced expression of CD94/NKG2A inhibitory receptors in human T lymphocytes. Eur. J. Immunol. 29:23-29.

46. Lohwasser, S., Kubota, A., Salcedo, M., Lian, R.H., and Takei, F. 2001. The non-classical MHC class I molecule Qa-1(b) inhibits classical MHC class Irestricted cytotoxicity of cytotoxic $T$ lymphocytes. Int. Immunol. 13:321-327.

47. Jia, S.H., Kurepa, Z., Bai, A., and Forman, J. 2000. Comparative ability of Qdm/Qa-1b, kb, and Db to protect class Ilow cells from NK-mediated lysis in vivo. J. Immunol. 165:6142-6147.

48. Anderton, S.M., van der, Z.R., Noordzij, A., and van Eden, W. 1994. Differential mycobacterial 65-kDa heat shock protein $\mathrm{T}$ cell epitope recognition after adjuvant arthritis-inducing or protective immunization protocols. J. Immunol. 152:3656-3664.

49. van Roon, J.A., van Eden, W., van Roy, J.L., Lafeber, F.J., and Bijlsma, J.W. 1997. Stimulation of suppressive $\mathrm{T}$ cell responses by human but not bacterial $60-\mathrm{kD}$ heat-shock protein in synovial fluid of patients with rheumatoid arthritis. J. Clin. Invest. 100:459-463.

50. Quintana, F.J., Carmi, P., Mor, F., and Cohen, I.R. 2002. Inhibition of adjuvant arthritis by a DNA vaccine encoding human heat shock protein 60 . J. Immunol. 169:3422-3428.

51. Quintana, F.J., Carmi, P., and Cohen, I.R. 2002. DNA vaccination with heat shock protein 60 inhibits cyclophosphamide-accelerated diabetes. J. Immunol. 169:6030-6035.

52. Rees, M.A., Rosenberg, A.S., Munitz, T.I., and Singer, A. 1990. In vivo induction of antigen-specific transplantation tolerance to Qa1a by exposure to alloantigen in the absence of T-cell help. Proc. Natl. Acad. Sci. U. S. A. 87:2765-2769.

53. Huston, D.P., Tavana, G., Rich, R.R., and Gressens, S.E. 1986. Regulation of primary cytotoxic T lymphocyte responses generated during mixed leukocyte culture with H-2d identical Qa-1-disparate cells. J. Immunol. 137:1776-1781.

54. Huston, D.P., and Tavana, G. 1987. Suppression of Ia-unrestricted primary anti-Qa-1 cytotoxic T lymphocyte responses by class II major histocompatibility complex-restricted cellular interactions. J. Immunol. 138:2753-2759.

55. Douillard, P., et al. 1999. Reassessment of the role of CD8+ T cells in the induction of allograft tolerance by donor-specific blood transfusion. Eur. J. Immunol. 29:1919-1924. 